\section{Utilisation des lymphocytes T régulateurs en transplantation}

\section{Supprimer l'immunosuppression}

Olivier Joffre, Thibault Santolaria, Joost P.M. van Meerwijk
0. Joffre: Inserm U563, Section Tolérance et Auto-immunité, 31300 Toulouse, France.

Adresse actuelle: CRUK, LRI, Immunobiology Lab, London WC2A 3PX, Royaume-Uni.

T. Santolaria : Inserm U563,

Section Tolérance et Auto-immunité,

31300 Toulouse, France.

J.P.M. van Meerwijk : Inserm U563,

Section Tolérance et Auto-immunité, 31300 Toulouse, France.

Université Paul Sabatier Toulouse III, 31400 Toulouse, France.

Institut Universitaire de France, Toulouse, France.

Joost.van-Meerwijk@inserm.fr

Olivier.Joffre@cancer.org.uk
La transplantation est une stratégie thérapeutique attractive confrontée à de nombreux obstacles. Le don d'organe est notamment limité par le sentiment ambivalent qu'il provoque, mêlé du désir de voir repoussées les limites de la vie et d'une profonde aversion à l'égard de la manipulation de la mort. D'un point de vue médical, la principale barrière est représentée par le système immunitaire du receveur qui met en place et coordonne un ensemble de mécanismes visant à détruire le greffon allogénique, considéré à juste titre comme du nonsoi. Pour contrôler les différentes formes de rejet, un large panel d'immunosuppresseurs a été développé depuis 40 ans. Conjugués à l'optimisation des techniques chirurgicales et des méthodes de conservation, ils ont permis de considérablement augmenter la survie des greffons (Figure 1). Ces molécules présentent cependant de nombreux inconvénients. Comme tout traitement médicamenteux, leur efficacité est influencée par des différences pharmacocinétiques interindividuelles. La large distribution tissulaire et la nature moléculaire de leurs cibles sont à l'origine d'effets iatrogènes majeurs, notamment au niveau des tissus chargés de l'épuration de l'organisme et/ou présentant un fort taux de renouvellement cellulaire. De plus, ces molécules inhibent globalement le système immunitaire et non spécifiquement le compartiment responsable du rejet. Cette immunosuppression générale altère les mécanismes d'immuno- surveillance, augmentant ainsi la fréquence de néoplasmes et d'infections et la morbidité qui leur est associée. Enfin, si elles sont efficaces pour lutter contre les épisodes de rejet aigu, ces drogues n'ont que peu d'effet sur le rejet chronique (Figure 1). Face à ces limitations majeures, la communauté scientifique tente de développer des stratégies visant à induire une tolérance au greffon, c'està-dire un état d'hyporéponse immunologique spécifique des alloantigènes. La majorité des approches envisagées à-vis des antigènes du donneur. Dès 1953, les travaux de Billingham, Brent et Medawar ont confirmé cette hypothèse [1]. Si leur approche basée sur l'induction d'un chimérisme hématopoïétique durant la vie fœtale apparaît inexploitable en clinique humaine, des études récentes suggèrent que des protocoles similaires sont envisageables chez des individus adultes [2].

repose sur un concept commun: détourner les mécanismes de tolérance au soi de leur fonction première.

La tolérance «au soi » est un processus nécessaire afin d'éviter que le système immunitaire ne s'attaque aux tissus de l'organisme. Elle est assurée par différents mécanismes complémentaires. Dans les organes lymphoïdes primaires, les précurseurs lymphocytaires autospécifiques sont éliminés après interaction avec des cellules d'origine hématopoïétique. Cette observation a conduit de nombreuses équipes à postuler qu'une greffe de moelle osseuse allogénique puisse induire un état de tolérance vis-

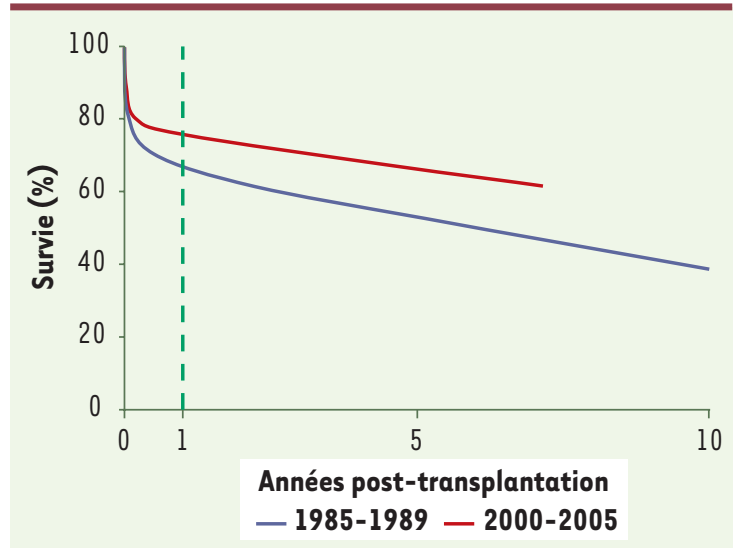

Figures 1. Survie des patients après transplantation cardiaque selon la période de greffe. L'augmentation significative de la médiane de survie des receveurs entre les périodes 1985-1989 et 1995-1999 (72,3 mois contre 131,9) est essentiellement associée au développement de stratégies thérapeutiques qui ont permis de contrôler efficacement les épisodes de rejet aigu et ainsi, d'augmenter la survie du greffon à court terme. Après la première année, entre 2 et $3 \%$ des patients perdent encore chaque année le greffon en raison du développement d'une forme de rejet qualifiée de chronique (source: Agence de la biomédecine, www.agence-biomedecine.fr). 
Un autre mécanisme impliqué dans l'induction et la maintenance de la tolérance «au soi » est dépendant de l'action de lymphocytes $T$ régulateurs dont l'existence, suspectée depuis les travaux de Le Douarin [3], a été définitivement établie par l'équipe de Sakaguchi [4]. L'observation que leur déplétion entraîne le développement d'une pathologie auto-immune létale [5] démontre que ces cellules, de phénotype $\mathrm{CD} 4^{+} \mathrm{CD} 25^{+}$Foxp $3^{+}$et naturellement produites dans le thymus, ont un rôle non-redondant dans le contrôle des lymphocytes autospécifiques [11]. Différents groupes ont développé des approches visant à évaluer leur utilisation potentielle dans le cadre de la transplantation.
Une nouvelle stratégie de contrôle du rejet de greffe de moelle osseuse par des $T$ régulateurs amplifiés

La capacité des lymphocytes T régulateurs à contrôler les mécanismes de rejet dépend du rapport de force entre les compartiments suppresseurs et effecteurs allospécifiques. Les cellules T régulatrices n'étant présentes dans l'organisme qu'en quantités limitées, les protocoles d'immunothérapie ont longtemps été bloqués par le faible nombre de cellules obtenues après purification. De plus, contrairement au compartiment des lymphocytes effecteurs, le répertoire des lymphocytes T régulateurs est pauvre en précurseurs allospécifiques [6]. Dans le laboratoire, nous avons levé ces deux barrières en développant une approche

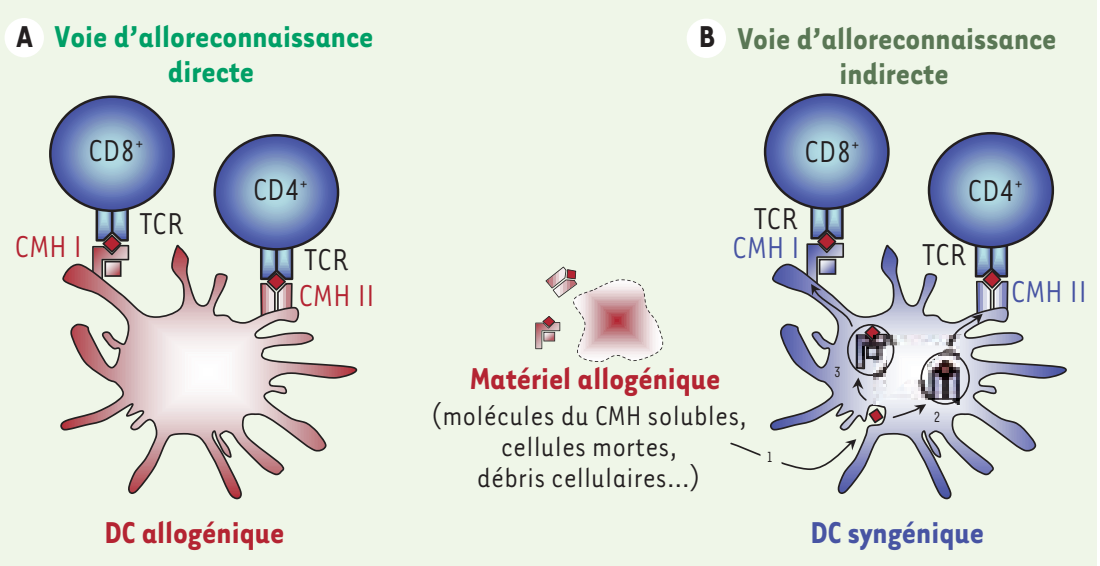

Figures 2. Deux voies d'alloreconnaissance conduisent à l'activation des lymphocytes T allospécifiques. A. Dans les semaines suivant l'opération, les cellules dendritiques (DC) allogéniques présentes dans le tissu greffé migrent dans les ganglions lymphatiques drainant. Elles vont alors stimuler les cellules T allospécifiques par une voie d'alloreconnaissance qualifiée de « directe » qui implique l'interaction du récepteur à l'antigène des lymphocytes T (TCR) avec les molécules de CMH allogéniques exprimées par les DC du donneur. Sauf dans le cas particulier de la greffe de moelle osseuse, le nombre de $D C$ allogéniques est fini puisque ces cellules ne sont pas renouvelées. La voie d'alloreconnaissance directe est donc principalement associée au rejet aigu. B. Les DC du receveur activent les cellules T allospécifiques par une voie « indirecte », essentiellement impliquée dans l'initiation et le développement du rejet chronique. Elle est dépendante de la présentation de peptides allogéniques par les molécules de $\mathrm{CMH}$ endogènes. Les antigènes du donneur, sous forme de cellules mortes ou de molécules solubles, sont captés par phagocytose ou pinocytose par les $D C$ du receveur (1). Ils sont alors dirigés vers la voie de biosynthèse des molécules du CMH II sur lesquelles ils sont chargés sous forme de peptides après protéolyse (2). Ce processus aboutit à l'activation des lymphocytes T CD4 ${ }^{+}$allospécifiques. Certaines populations de $D C$ sont aussi capables de présenter, par un mécanisme de cross-présentation, les antigènes exogènes allogéniques aux cellules T CD8 ${ }^{+}$via les molécules du CMH I (3). qui nous a permis de prévenir le rejet d'une allogreffe de moelle osseuse [7]. Après purification, nous avons augmenté le nombre de lymphocytes T régulateurs issus du receveur via une étape de culture in vitro. Afin d'enrichir la population obtenue en précurseurs allospécifiques, nous avons utilisé des cellules présentatrices d'antigènes du donneur comme source de signal mitogène. Nous avons alors testé la capacité des cellules T régulatrices du receveur ainsi obtenues à contrôler les mécanismes de rejet après allogreffe de moelle osseuse chez des animaux conditionnés par une irradiation non-lymphoablative. Le but avoué du prétraitement était de libérer des niches pour les cellules souches hématopoïétiques allogéniques et de déprimer transitoirement et partiellement la réactivité du système immunitaire afin de faciliter l'action de la population suppressive injectée. Dans ce système, nous avons montré que les cellules régulatrices issues du receveur et amplifiées selon notre protocole contrôlent de façon durable le rejet dirigé contre la moelle osseuse allogénique. Contrairement aux drogues immunosuppressives, nous avons observé que ce traitement inhibe uniquement le compartiment alloréactif, et non le système immunitaire dans sa globalité. Notre procédure permet donc un contrôle total du rejet tout en évitant les effets secondaires des drogues. L'intérêt de ce type d'approche repose aussi sur le fait qu'à leur tour, les cellules hématopoïétiques allogéniques transférées participent à l'induction de tolérance des lymphocytes T et $B$ néosynthétisés dans les organes lymphoïdes primaires ainsi qu'à la survie de la population régulatrice injectée.

Encouragés par ces résultats, nous avons utilisé le même protocole pour tenter de prévenir le rejet de tissus ou d'organes solides [8]. Contrairement aux résultats obtenus après allogreffe de moelle osseuse, les cellules $T$ régulatrices n'ont que légèrement retardé la destruction de greffes allogéniques de cœur ou de peau. Étant donné que les lymphocytes T régulateurs ne survivent qu'en présence 
des antigènes pour lesquels ils sont spécifiques, et que contrairement à la moelle osseuse, les tissus solides n'ont que peu de cellules présentatrices d'antigènes capables de délivrer les signaux nécessaires, nous avons testé si la co-injection de moelle osseuse allogénique avec les cellules $T$ régulatrices pourrait favoriser la protection de la greffe solide. Dans ce contexte, les lymphocytes $T$ régulateurs préviennent donc le rejet de la moelle qui en retour favorise leur survie. Cette approche nous a permis d'inhiber efficacement les épisodes de rejet aigu mais pas le développement du rejet chronique. Ce résultat démontre qu'un chimérisme hématopoïétique ne garantit pas la survie des greffes à long terme.

\section{Une préactivation \\ des cellules $\mathrm{T}$ régulatrices \\ par les voies d'alloreconnaissance \\ directes et indirectes est nécessaire pour inhiber totalement les mécanismes de rejet aigu et chronique}

Le rejet d'allogreffe est orchestré par des lymphocytes $T$ susceptibles de reconnaître les alloantigènes de deux façons fondamentalement distinctes (Figure 2). À court terme, les cellules $T$ allospécifiques sont stimulées «directement» par les cellules présentatrices d'antigène du donneur migrant du greffon aux organes lymphoïdes draînants. Dans ce cas, les cellules T de l'hôte reconnaissent donc les peptides allogéniques présentés par les molécules $\mathrm{du}$ CMH du donneur. Cette voie d'activation est essentiellement associée aux épisodes de rejet aigu. À plus long terme, les cellules présentatrices d'antigène de l'hôte colonisent le tissu greffé, captent des antigènes, avant d'activer «indirectement » d'autres lymphocytes T allospécifiques. Ces derniers, qui reconnaissent donc des complexes formés par des molécules du CMH du soi et des peptides allogéniques, vont alors initier les mécanismes de rejet chronique. Le protocole de culture que nous avions établi ne permettait d'enrichir la population régulatrice qu'en cellules reconnaissant les antigènes du donneur par la voie directe. Nous avons émis l'hypothèse selon laquelle l'absence de contrôle des mécanismes de rejet chronique était due à l'absence, dans la population injectée, de cellules suppressives reconnaissant les alloantigènes par voie indirecte. Nous avons donc modifié notre protocole de culture in vitro afin de générer une population de cellules T régulatrices enrichie en précurseurs capables de reconnaître les alloantigènes à la fois par les voies directe et indirecte. En utilisant cette population, nous avons pu inhiber totalement les mécanismes de rejet chronique et ainsi protéger à long terme des doubles greffes de moelle osseuse et de peau ou de cœur. En développant une stratégie permettant de dévier les deux mécanismes d'induction de tolérance au soi de leur fonction première, nous avons donc induit un état de tolérance durable et spécifique à des allogreffes de tissus et d'organes.

\section{Sur le chemin}

d'une utilisation clinique?

Si ces données sont encourageantes, de nombreux obstacles restent cependant à résoudre avant de transposer notre approche à la clinique humaine. Le premier est logistique puisqu'il conviendra de contrôler le rejet de la moelle et de l'organe le temps nécessaire à la culture des cellules T régulatrices. Le deuxième concerne le fait que les lymphocytes T mémoires jouent un rôle important dans le rejet de greffe chez I'homme. Les souris de laboratoire étant élevées et maintenues dans des structures exemptes de pathogènes, leur système immunitaire en est quasiment dépourvu. Des travaux complémentaires sont donc nécessaires afin d'évaluer la capacité des cellules T régulatrices à contrôler l'alloréactivité des lymphocytes mémoires. Le troisième obstacle est lié à la purification des cellules régulatrices. Chez des animaux de laboratoire, un enrichissement basé sur l'expression du marqueur CD25 permet d'obtenir une population virtuellement pure de cellules suppressives. Chez I'homme, la situation est plus complexe puisque le marqueur CD25 est exprimé par les cellules T activés et que Foxp3, de par sa localisation nucléaire, n'est pas exploitable. Des études récentes semblent suggérer que d'autres marqueurs, tels que CD127 ou FR4, pourraient être utilisés [9, $10]$.

Même si la route est encore longue, notre travail suggère que les cellules $T$ régulatrices pourront être utilisées en clinique afin d'induire un état de tolérance à un greffon allogénique. Le bénéfice attendu pour les patients est immense puisque ce type d'approche permettra de contrôler totalement et durablement les mécanismes de rejet sans induire de toxicité et sans favoriser le développement de néoplasmes ou d'infections opportunistes. $\diamond$

Tregs-based immunotherapy: an efficient way to fully inhibit acute and chronic rejection

\section{RÉFÉRENCES}

1. Billingham RE, Brent L, Medawar PB. Actively acquired tolerance of foreign cells. Nature 1953; 172: 603-6.

2. Kawai T, Cosimi AB, Spitzer TR, et al. HLA-mismatched renal transplantation without maintenance immunosuppression. N Engl J Med 2008 ; 358 : 353-61.

3. Coutinho A, Salaun J, Corbel C, et al. The role of thymic epithelium in the establishment of transplantation tolerance. Immunol Rev 1993; 133 : 225-40.

4. Sakaguchi S, Sakaguchi N, Asano M, et al. Immunologic self-tolerance maintained by activated T cells expressing IL-2 receptor alpha-chains (CD25). Breakdown of a single mechanism of self-tolerance causes various autoimmune diseases. I Immunol 1995 ; 155 : 1151-64.

5. Sakaguchi S. Naturally arising $\mathrm{CD} 4^{+}$regulatory T cells for immunologic self-tolerance and negative control of immune responses. Annu Rev Immunol 2004; $22: 531-62$.

6. Romagnoli P, Hudrisier D, van Meerwijk JPM. Preferential recognition of self antigens despite normal thymic deletion of $\mathrm{CD}^{+} \mathrm{CD} 25^{+}$regulatory $\mathrm{T}$ cells. J Immunol 2002 ; 168 : 1644-8.

7. Joffre 0 , Gorsse $N$, Romagnoli P, et al. Induction of antigen-specific tolerance to bone marrow allografts with CD4+CD25+ T lymphocytes. Blood 2004 ; $103: 4216-21$.

8. Joffre 0 , Santolaria T, Calise D, et al. Prevention of acute and chronic allograft rejection with CD4+CD25+Foxp3+ regulatory T lymphocytes. Nat Med $2008 ; 14: 88-92$.

9. Liu W, Putnam AL, Xu-Yu Z, et al. CD127 expression inversely correlates with FoxP3 and suppressive function of human CD4 ${ }^{+}$T reg cells. J Exp Med 2006; 203 : 1701-11.

10. Yamaguchi T, Hirota K, Nagahama K, et al. Control of immune responses by antigen-specific regulatory $T$ cells expressing the folate receptor. Immunity 2007 ; $27: 145-59$.

11. Vocanson M, Hennino A, Chavagnac C, et al. Eczéma allergique de contact : comment ré-induire une tolérance? Med Sci (Paris) 2006 ; 22 : 158-63. 